\title{
Hands-on gravitational wave astronomy: Extracting astrophysical information from simulated signals
}

\author{
Louis J. Rubbo \\ Pennsylvania 16802 \\ Shane L. Larson ${ }^{\text {a) }}$ \\ Department of Physics, Weber State University, Ogden, Utah 84408 \\ Michelle B. Larson ${ }^{\text {a) }}$ \\ Department of Physics, Utah State University, Logan, Utah 84322 \\ Dale R. Ingram \\ LIGO Hanford Observatory, Richland, Washington 99352
}

Center for Gravitational Wave Physics, Pennsylvania State University, University Park,

(Received 4 October 2006; accepted 9 March 2007)

\begin{abstract}
In this article we introduce an activity in which introductory astronomy students act as gravitational wave astronomers by extracting information from simulated gravitational wave signals. The process mimics the way real gravitational wave analysis is handled by using plots of a pure gravitational wave signal. The students measure the properties of the simulated signal and use these measurements to evaluate standard relations for astrophysical source parameters. Although the activity described focuses on circular binaries, the techniques described can be applied to other gravitational wave sources as well. A problem based on the discussion in this paper is available for use in introductory laboratory courses. (C) 2007 American Association of Physics Teachers.
\end{abstract}

[DOI: $10.1119 / 1.2721587]$

\section{INTRODUCTION}

Observational astronomy is at the threshold of an era where gravitational wave detectors regularly contribute important information to the growing body of astrophysical knowledge. ${ }^{1}$ Ground based detectors such as the Laser Interferometer Gravitational-wave Observatory ${ }^{2}$ (LIGO) and the forthcoming space based detector, the Laser Interferometer Space Antenna ${ }^{3}$ (LISA), will probe different regimes of the gravitational wave spectrum and observe sources that radiate at different gravitational wavelengths. Unlike traditional electromagnetic telescopes, gravitational wave detectors are not imaging instruments. How then does a gravitational wave astronomer take the output from a detector and extract astrophysical information about the emitting sources? This paper introduces a hands-on activity in which introductory astronomy students answer this question.

Traditional astronomy is usually presented through the medium of colorful images taken with large scale telescopes. In addition to studying images, astronomers learn about astrophysical systems by collecting data at multiple wavelengths using, for example, narrow-band spectra and measuring time-varying light curves. The core physics governing the evolution of these distant systems is often deduced from the physical character of the observed electromagnetic radiation, rather than from the imagery that is used to illustrate the science.

Gravitational wave astronomy is analogous to its electromagnetic cousin, with one important distinction: there is no image data. Gravitational wave observatories like LIGO and LISA return a noisy time series that has encoded within it gravitational wave signals from one or possibly many overlapping sources. To gain information about the systems emitting these gravitational wave signals requires the use of time series analysis techniques such as Fourier transforms, Fisher information matrices, and matched filtering. Recently, it was shown in Ref. 4 how students can emulate the match filtering process by comparing ideal signals to mocked noisy detector output. ${ }^{4}$ In this paper, a procedure is described in which students can analyze a simulated gravitational wave signal and extract the astrophysical parameters that describe the radiating system. The goal is to introduce students to how gravitational wave astronomers learn about sources of gravitational radiation.

Section II outlines the theory relating the structure of gravitational waves to astrophysical parameters, and Sec. III illustrates the characteristic waveforms from a typical binary system. Section IV illustrates a procedure where measurements made from waveform plots, together with the theory of waveform generation, can be used to extract the astrophysical parameters of the system emitting gravitational radiation. Section V discusses implementations and extensions of this activity in an introductory astronomy course. The analysis described in the paper has been implemented in an activity format, complete with a keyed solution for the instructor, and is publicly available. ${ }^{5}$

\section{GRAVITATIONAL WAVE PRODUCTION IN BINARIES}

In electromagnetism radiation is produced by an accelerating charged particle. Similarly, in general relativity, gravitational radiation is produced by an accelerating mass. More precisely, gravitational waves are produced by a time varying mass quadrupole moment. ${ }^{6}$ Monopole radiation is prevented due to conservation of mass, and dipole radiation does not occur due to the conservation of momentum. Hence, the quadrupole is the leading order term in the multipole expansion of the radiation field. Expected astrophysical examples of time varying quadrupole moments include spinning neutron stars with surface deformities, the inspiral and eventual 
plunge of compact objects into massive black holes, and asymmetric supernovae. ${ }^{7}$ This paper focuses on an additional example, a binary star system.

For a circular binary system, where the components are treated as point-like particles, the gravitational waveforms take on the seductively simple form

$$
h(t)=\mathcal{A}(t) \cos \Phi(t)
$$

where $h(t)$ is the gravitational waveform (also referred to as the gravitational wave strain), $\mathcal{A}(t)$ is the time dependent amplitude, and $\Phi(t)$ is the gravitational wave phase. The amplitude $\mathcal{A}(t)$ can be expressed in terms of the parameters characterizing the system,

$$
\mathcal{A}(t)=\frac{2(G \mathcal{M})^{5 / 3}}{c^{4} r}\left(\frac{\pi}{P_{\mathrm{gw}}(t)}\right)^{2 / 3},
$$

where $G$ is Newton's gravitational constant, $c$ is the speed of light, $r$ is the luminosity distance to the binary, and $P_{\mathrm{gw}}(t)$ is the gravitational wave period. The quantity $\mathcal{M}$ $\equiv\left(M_{1} M_{2}\right)^{3 / 5}\left(M_{1}+M_{2}\right)^{-1 / 5}$ is the chirp mass and appears often in gravitational wave physics, making it a natural mass scale. The origin for this nomenclature will become evident shortly. The gravitational wave phase, $\Phi(t)$, is analogous to the phase for other wave phenomenon. It represents the location of the wave cycle which is impinging on the detector at time $t$, and tracks the evolution of the wave's amplitude as a function of time. The phase has units of radians, and is related to the gravitational wave frequency $f_{\mathrm{gw}}$ (or, alternatively, the period $P_{\mathrm{gw}}=f_{\mathrm{gw}}^{-1}$ ) by the integral,

$$
\Phi(t)=\Phi_{0}+2 \pi \int_{0}^{t} \frac{d t^{\prime}}{P_{\mathrm{gw}}\left(t^{\prime}\right)} .
$$

Here $\Phi_{0}$ is the initial phase value, $\Phi_{0}=\Phi(t=0)$.

If we return to the amplitude given in Eq. (2) and substitute typical values for a neutron star binary located at the center of our galaxy, we find that gravitational waves are extremely weak,

$$
\mathcal{A}=10^{-22}\left(\frac{\mathcal{M}}{1.22 \mathrm{M}_{\odot}}\right)^{5 / 3}\left(\frac{r}{8 \mathrm{kpc}}\right)^{-1}\left(\frac{P_{\mathrm{gw}}}{10^{3} \mathrm{~s}}\right)^{-2 / 3}
$$

An analysis of the units in Eq. (2) reveals that the amplitude $\mathcal{A}$ is dimensionless. Gravitational radiation manifests itself in matter by inducing a strain. In other words, Eq. (1) gives the relative change in the distance between two points in spacetime as a function of time. By monitoring the separation between two or more test masses, it is possible to discern if a gravitational wave is present. The measurement of the gravitational radiation field directly is in contrast to most electromagnetic observations where the energy flux (the square of the field) is measured.

As in electromagnetism, gravitational waves have two independent polarization states. For a binary system the two states are related by a $90^{\circ}$ phase shift. Consequently, Eq. (1) captures the functional form for both polarization states. For the purposes of this paper only a single polarization state and its associated waveform will be discussed.

Gravitational waves carry energy and angular momentum away from the binary system causing the orbital period to decrease with time according to ${ }^{8}$

$$
P_{\text {orb }}(t)=\left(P_{0}^{8 / 3}-\frac{8}{3} k t\right)^{3 / 8},
$$

where $P_{0}$ is the orbital period at time $t=0$, and $k$ is an evolution constant given by

$$
k \equiv \frac{96}{5}(2 \pi)^{8 / 3}\left(\frac{G \mathcal{M}}{c^{3}}\right)^{5 / 3}
$$

As a result of the decreasing orbital period, the two binary components will slowly inspiral, eventually colliding and coalescing into a single remnant. For the assumption of point-like particles, the coalescence occurs when $P_{\text {orb }}(t)=0$.

Although gravitational waves have not yet been detected directly, they were first indirectly detected through orbital period decay in the early 1970s when Hulse and Taylor observed the first known binary pulsar, PSR $1913+16$. $^{9} \mathrm{Be}-$ cause pulsars are extremely accurate clocks, it was possible to use changes in the pulse intervals to measure the changing orbital period as the system decays. The orbital decay rate was found to agree with general relativity's prediction of gravitational radiation to exquisite accuracy. ${ }^{10}$

It is important to emphasize that Eq. (5) gives the orbital period, not the gravitational wave period. Careful scrutiny of Eqs. (2) and (3) reveals that the gravitational wave period $P_{\mathrm{gw}}(t)$ appears in the description of the waveform. For circularized binary systems, $P_{\mathrm{orb}}(t)$ and $P_{\mathrm{gw}}(t)$ are simply related by

$$
P_{\mathrm{orb}}(t)=2 P_{\mathrm{gw}}(t)
$$

The factor of two stems from the fact that the lowest possible order for gravitational radiation production is the quadrupole order. Quadrupole moments are invariant under a $180^{\circ}$ rotation, yielding a factor of two per complete orbit.

\section{WAVEFORMS FROM A BINARY SYSTEM}

As an example of the kind of waveforms we expect from binaries, consider a binary neutron star system with $M_{1}$ $=M_{2}=1.4 \mathrm{M}_{\odot}\left(\mathcal{M}=1.22 \mathrm{M}_{\odot}\right)$ located at the center of the galaxy $r=8 \mathrm{kpc}$ away. We will consider waveforms generated at two distinct times in the binary's evolution. The first waveform that we will consider is $\sim 10^{6}$ years before coalescence. During this phase the gravitational wave frequency is detectable by the spaceborne LISA observatory, which is sensitive to radiation between $10^{-5}$ and $1 \mathrm{~Hz}$. The second waveform we will consider is during the final second before the neutron star binary coalesces. The gravitational wave frequencies during this phase are detectable by the terrestrial LIGO observatory, which is sensitive to gravitational wave frequencies between 64 and $2000 \mathrm{~Hz}$.

\section{A. Far from coalescence}

Figure 1 shows the emitted gravitational radiation long before the binary components coalesce. The figure was produced using Eqs. (1)-(7) and the values given previously. During this era of the binary evolution, the gravitational waves are essentially monochromatic; the orbital period is evolving too slowly to detect a frequency derivative term. For such monochromatic signals the only measurable properties of the gravitational waveform are the period $P_{\mathrm{gw}}$ [and the orbital period $P_{\text {orb }}$ through Eq. (7)], the amplitude $\mathcal{A}$, and the initial phase $\Phi_{0}$. Even though the waveform equations depend on the chirp mass $\mathcal{M}$ and the luminosity distance $r$, it 


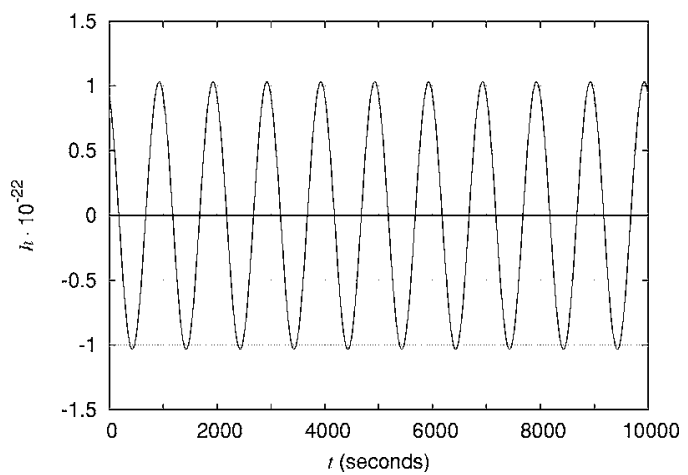

Fig. 1. The gravitational waveform for a binary system consisting of two neutron stars far from coalescence and located at the center of our galaxy.

is not possible to solve for their values from the data provided by the monochromatic waveform. Not enough information exists to completely solve Eqs. (2) and (5) for both quantities. The inability to detect a change in the orbital period can be seen by considering the relative size of the two terms in Eq. (5). If we use the binary neutron star chirp mass $\mathcal{M}=1.22 \mathrm{M}_{\odot}$, it is evident that the second term is negligible compared to the period $P_{0}$ of the wave shown in Fig. 1. In the parlance of gravitational wave astronomy, there is a mass-distance degeneracy in the waveform description, analogous to the familiar mass-inclination degeneracy in the electromagnetic observations of spectroscopic binaries. ${ }^{11}$ This degeneracy is well known, but we will show that it can be broken if the orbital period of the binary evolves during the gravitational wave observations.

\section{B. Near coalescence}

Inspection of Eq. (5) shows that, as time goes on, the emission of gravitational waves causes the orbital period to become shorter and, as a result, the frequency of the emitted waves increases. Similarly, consideration of Eq. (2) shows that as the wave period decreases, the time dependent amplitude $\mathcal{A}(t)$ increases. These changes are characteristic of gravitational waves emitted just prior to a source coalescence and are referred to collectively as a chirp signal. The chirp waveform emitted by the example binary neutron star system just prior to coalescence is illustrated in Fig. 2.

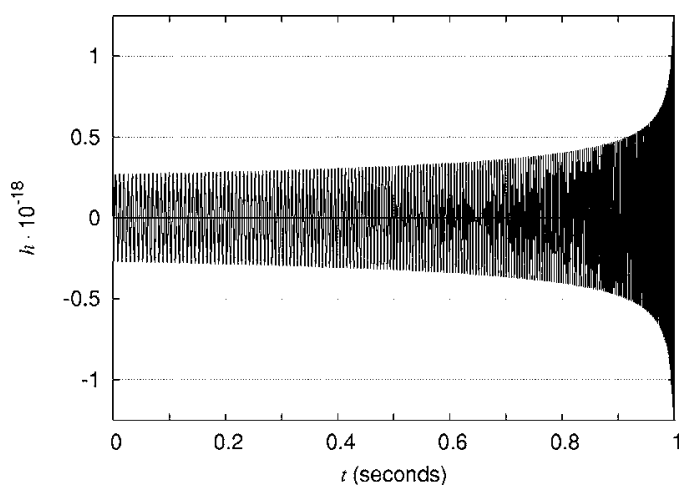

Fig. 2. The waveform over the last second before coalescence. Because the signal's amplitude and frequency increase with time, these types of systems are said to be chirping.

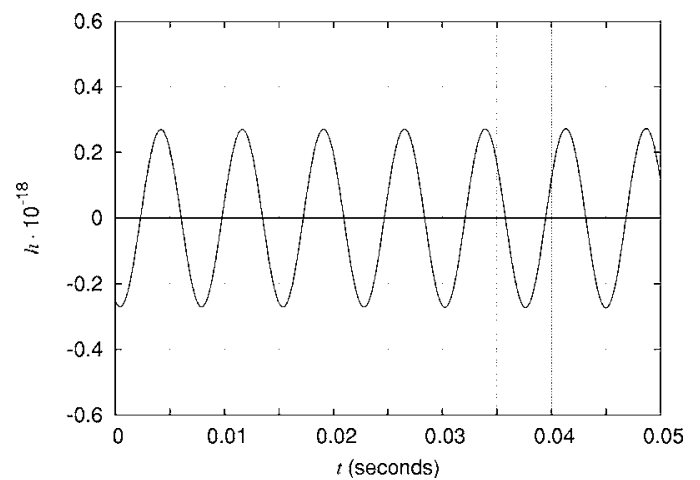

Fig. 3. The chirping waveform one second before coalescence.

A binary signal that evolves appreciably during the gravitational wave observation is called a chirping binary. In these cases, the mass parameter $\mathcal{M}$ which appears in the amplitude $\mathcal{A}(t)$ and in the period evolution constant $k$ can be determined from measurements of the evolving signal. For this reason, the mass $\mathcal{M}$ is called the chirp mass. To leading order in the gravitational wave production, it is not possible to measure the individual masses, only the chirp mass. Consequently, it is not possible to distinguish between binaries with the same chirp mass. For example, the binary neutron star considered here with $M_{1}=M_{2}=1.4 \mathrm{M}_{\odot}$ has roughly the same chirp mass as a binary with an $M_{1}=10 \mathrm{M}_{\odot}$ black hole and a $M_{2}=0.3 \mathrm{M}_{\odot}$ white dwarf.

To extract the chirp mass from measurements of the gravitational waveform, consider two small stretches of the chirping waveform. Figure 3 shows the waveform from $0 \mathrm{~s} \leqslant t$ $\leqslant 0.05 \mathrm{~s}$, and Fig. 4 shows the waveform from $0.9 \mathrm{~s} \leqslant t$ $\leqslant 0.92 \mathrm{~s}$. The waveform is appreciably different between these two snapshots, both in amplitude $\mathcal{A}(t)$ and in period $P_{\mathrm{gw}}(t)$. This difference allows the degeneracy found in the monochromatic signal case to be broken, because the gravitational wave period can be measured at two different times and used in Eq. (5) to solve for the chirp mass $\mathcal{M}$.

\section{MEASURING GRAVITATIONAL WAVEFORMS}

This section illustrates a procedure where introductory astronomy students can make measurements from the figures in Sec. III using a straight edge. From their measured data

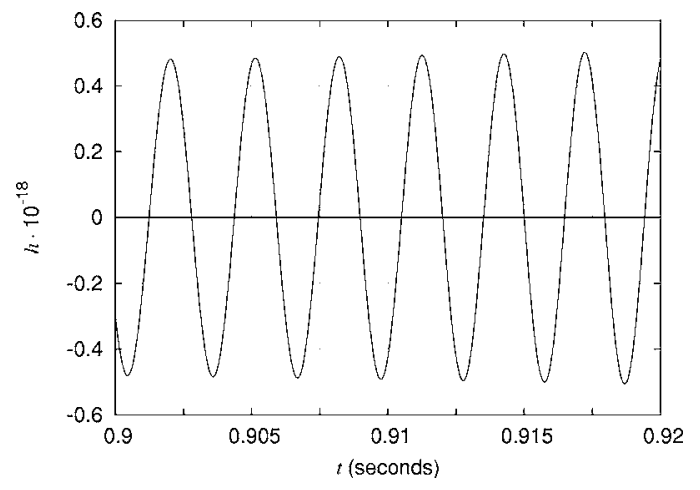

Fig. 4. The chirping waveform one-tenth of a second before coalescence. 
and the theoretical results in Sec. II they can deduce the astrophysical character of the system emitting the gravitational waveforms.

\section{A. Monochromatic waveforms}

Limited astrophysical information can be extracted directly from Fig. 1, as will be the case with true monochromatic signals detected by gravitational wave observatories. With limited assumptions more detailed information can be deduced. A suitable extraction and analysis procedure for an introductory astronomy student would proceed in the following manner:

(1) Measure the gravitational wave period $P_{\mathrm{gw}}$ from Fig. 1. Because the signal is monochromatic and the binary is circular, the orbital period $P_{\text {orb }}$ is obtained from $P_{\mathrm{gw}}$ using Eq. (7). For the waveform in Fig. 1, a careful measurement should yield $P_{\mathrm{gw}}=1000 \mathrm{~s}$.

(2) Measure the maximum value for $h(t)$ shown in Fig. 1. This is the value for the amplitude $\mathcal{A}$ given in Eq. (2). As noted in Sec. III A, no astrophysical information can be extracted from the amplitude alone. When a binary system is far from coalescence, it is not possible to detect a changing orbital period. From Eq. (2) the same can be said about the amplitude. Therefore, the amplitude measured here can be taken to be time-independent.

(3) Measure the initial phase for the waveform displayed in Fig. 1. This is done by noting that at $t=0$ the gravitational waveform reduces to

$h(t=0)=A \cos \Phi_{0}$,

which in turn implies that

$\Phi_{0}=\cos ^{-1}\left(\frac{h(t=0)}{A}\right)$.

For the plots in Fig. 1, a careful measurement should give a value near $\Phi_{0}=\pi / 7 \approx 0.45$. The initial phase does not represent an intrinsic property of the binary; its value is a consequence of when the gravitational wave observations began. To illustrate this property of the initial phase, imagine relabeling the time axis in Fig. 1 to represent a new observation that started later than the observation shown. The initial phase will have a new value, but the waveform does not change because the intrinsic properties of the binary did not change.

(4) If a gravitational wave astronomer were to assume that the binary was a pair of neutron stars, the component mass values could be assigned as part of the assumption. Most neutron star masses cluster around $M=1.4 \mathrm{M}_{\odot}$. A good initial assumption, and one that we will make from this point on, is that each component of the binary has this mass. As noted in Sec. III A, this assumption can be dangerous because similar chirp masses, $\mathcal{M}$, can result from significantly different systems. Additional information not present in the gravitational waveform might help an astronomer be more confident about such an assumption. For example, an associated simultaneous electromagnetic signal or the location of the source on the sky may favor one model of the binary over another. With the assumed component masses, the orbital separation $R$ of the binary components can be computed from the measured orbital period by using Kepler's third law,

$G\left(m_{1}+m_{2}\right)=\left(\frac{2 \pi}{P_{\text {orb }}}\right)^{2} R^{3}$

For this example, the orbital separation is $R=1.4$ $\times 10^{-3} \mathrm{AU}=2.1 \times 10^{8} \mathrm{~m}$, a little less than the separation of the Earth and the Moon.

(5) If the masses are assumed, the distance to the binary can be calculated from Eq. (2) and the measured amplitude. If careful measurements have been made, the answer should be close to the value $r=8 \mathrm{kpc}=2.5 \times 10^{20} \mathrm{~m}$.

(6) Finally, if the masses are assumed, it can be shown that the monochromatic description is a good one for this wave by computing the value of the second term in Eq. (5) and showing that it is negligible compared to the measured period $P_{0}$. The second term in Eq. (5) is found by using the assumed mass values to get the chirp mass and the final time shown on the plot for the value of $t$.

\section{B. Chirping waveforms}

For a chirping waveform, additional astrophysical information associated with the system can be extracted directly from measurements of the waveform without making underlying assumptions like those needed when the system is far from coalescence. To extract information from the chirping waveform shown in Fig. 2, the two zoom-ins of the waveform shown in Figs. 3 and 4 will be used. A typical extraction procedure might look like the following:

(1) For Figs. 3 and 4, measure the period of one cycle of the wave and note the time, $t$, at which the periods were measured. The amplitudes $\mathcal{A}(t)$ should be measured for the same cycle as the periods.

(2) If the period measured at time $t_{1}$ in Fig. 3 is $P_{0}$, and the period measured at time $t_{2}$ in Fig. 4 is $P_{\mathrm{gw}}(t)$ at time $t$ $=t_{2}-t_{1}$, then Eq. (5) can be used to deduce the chirp mass, $\mathcal{M}$, of the system.

(3) Once the chirp mass $\mathcal{M}$ has been determined, the distance to the binary can be computed by using Eq. (2) with the measured amplitude $\mathcal{A}(t)$ and period $P_{\mathrm{gw}}(t)$ of each waveform. The results from the two figures can be averaged together to obtain a final result for the distance to the binary.

\section{DISCUSSION}

The activity described here introduces how gravitational wave astronomers extract astrophysical information from observed binary waveforms. Real signal analysis is more complex. The most significant challenge for real data analysis is identifying the signal buried in a noisy data stream. A common approach to this problem in gravitational wave astronomy is to use template matching, which has been explored in a separate activity. ${ }^{4}$ If a signal is present in a noisy data stream, the template provides a way to subtract the noise and leave a clean waveform. This activity assumes a signal template has been selected. Astronomers estimate the values of astrophysical parameters describing a source of gravitational waves from clean waveforms.

We have introduced the core calculations in gravitational wave astrophysics that an introductory astronomy student can perform in a laboratory setting to gain information about 
an astrophysical system. To complement this article we have developed a detailed student activity sheet and corresponding teacher's guide in Sec. IV. The complementary material is available at Ref. 5.

\section{ACKNOWLEDGMENTS}

This work was supported by the Center for Gravitational Wave Physics, which is funded by the National Science Foundation under cooperative agreement PHY-01-14375. The authors would like to thank the LIGO Laboratory. The LIGO Observatories were constructed by the California Institute of Technology and Massachusetts Institute of Technology with funding from the National Science Foundation under cooperative agreement PHY-9210038. The LIGO Laboratory operates under cooperative agreement PHY0107417. This paper has been assigned LIGO Document Number LIGO-P060064-00-Z.

${ }^{a)}$ Formerly at the Center for Gravitational Wave Physics, Pennsylvania State University, University Park, Pennsylvania 16802.

${ }^{1}$ L. J. Rubbo, S. L. Larson, M. B. Larson, and K. D. Zaleski, "Gravita- tional waves: New observatories for new astronomy," Phys. Teach. 44, 420-423 (2006).

${ }^{2}$ A. Abramovici et al., "LIGO: The laser interferometer gravitational-wave observatory," Science 256, 325-333 (1992).

${ }^{3}$ T. J. Sumner and D. N. A. Shaul, "The observations of gravitational waves from space using LISA,” Mod. Phys. Lett. A 19, 785-800 (2004).

${ }^{4}$ M. B. Larson, L. J. Rubbo, K. D. Zaleski, and S. L. Larson, "Science icebreaker activities: An example from gravitational wave astronomy," Phys. Teach. 44, 416-419. (2006).

${ }^{5}\langle$ cgwp.gravity.psu.edu/outreach/activities/>.

${ }^{6}$ B. F. Schutz, "Gravitational waves on the back of an envelope," Am. J. Phys. 52, 412-419 (1984).

${ }^{7}$ C. Cutler and K. S. Thorne, "An overview of gravitational-wave sources," in General Relativity and Gravitation: Proceedings of the 16th International Conference, edited by N. T. Bishop and S. D. Maharaj (World Scientific, 2002), p. 72.

${ }^{8}$ J. B. Hartle, Gravity: An Introduction to Einstein's General Relativity (Addison-Wesley, San Francisco, 2003), p. 508.

${ }^{9}$ R. A. Hulse, "The discovery of the binary pulsar," Rev. Mod. Phys. 66, 699-710 (1994).

${ }^{10}$ J. H. Taylor Jr., "Binary pulsars and relativistic gravity," Rev. Mod. Phys. 66, 711-719 (1994).

${ }^{11}$ B. W. Carroll and D. A. Ostlie, An Introduction to Modern Astrophysics (Addison-Wesley, San Francisco, 2007), 2nd ed., Chap. 7.

\section{MAKE YOUR ONLINE MANUSCRIPTS COME ALIVE}

A picture is worth a thousand words. Film or animation can be worth much more. If you submit a manuscript which includes an experiment or computer simulation, why not make a film clip of the experiment or an animation of the simulation, and place it on EPAPS (Electronic Physics Auxiliary Publication Service). Your online manuscript will have a direct link to your EPAPS webpage.

See http://www.kzoo.edu/ajp/EPAPS.html for more information. 